\title{
Lingura
}

\section{Satzbegriff und gesprochene Sprache}

\author{
Walther Kindt \\ Fakultät für Linguistik und Literaturwissenschaft, Universität Bielefeld
}

Empfangen Oktober 1993

\section{Zusammenfassung}

Der Anspruch der modernen Linguistik, eine theoretisch fundierte empirische Wissenschaft zu sein, macht es notwendig, Lösungen für bestimmte notorische Grundlagenprobleme zu finden. Hierzu gehört auch die Frage einer angemessenen Definition des Satzbegriffs. Im vorliegenden Aufsatz wird gezeigt, wie man einen geeigneten empirisch operationalisierten und weitgehend grammatikunabhängigen Satzbegriff einführen kann. Zugleich ergibt sich, daß dieser Satzbegriff auch die Schwierigkeiten behebt, die man bei einer Segmentierung von Äußerungen der gesprochenen Sprache z.B. mit Ellipsen und Reparaturen hat.

\begin{abstract}
The claim of modern linguistics to be part of theoretically founded empirical sciences makes it necessary to look for solutions to certain notorious basic problems. This also implies the question of an adequate definition of the notion of sentence. The present paper shows how it is possible to introduce an adequate notion of sentence that is empirically operationalized and largely syntax-independent. From this also follows that this notion of clause will be able to take off the difficulties that arise when segmenting utterances of the spoken language, e.g. with ellipses and repairs.
\end{abstract}

\section{Einleitung}

Da Sätze ein zentraler Forschungsgegenstand der Linguistik sind, ist es nicht verwunderlich, daß man sich seit jeher bemüht hat, den vorverständlichen Satzbegriff zu explizieren und zu einer allgemeinverbindlichen Satzdefinition zu gelangen. Erstaunlich ist allerdings, wie aporetisch Diskussionen über den Satzbegriff auch heute noch ausgehen. So fand unlängst am Institut für Deutsche Sprache in Mannheim eine Podiumsdiskussion mit prominenten Vertretern der Linguistik statt (vgl. Hoffmann, 1992), die - polemisch gesagt - alles andere als Klarheit und Konsens über den Satzbegriff erbrachte. Nun ist es keineswegs so, daß es zu wenige Vorschläge für cine Explikation des Satzbegriffs gäbe. ${ }^{1}$ Vielmehr fehlt eine klare Posi-

1 Beispielsweise hat schon Seidel (1935) 220 verschiedene Satzdefinitionen gezählt. 
tion zum theoretischen Stellenwert und zur empirischen Brauchbarkeit der vielen vorliegenden Vorschläge. Dies bedeutet - und hierauf werde ich genauer eingehen daß das ungelöste Problem der Satzdefinition auch mit einer unzureichenden wissenschaftstheoretischen Diskussion zusammenhängt. Die moderne Wissenschaftstheorie hat sich derart konkreter Probleme linguistischer Theoriebildung nie angenommen, und insofern ist es verständlich, daß die Linguisten zwischen zwei polaren Positionen schwanken: einerseits der Hoffnung, man werde noch eine generell akzeptierte Satzdefinition finden, und andererseits die durch die bisherigen Mißerfolge begründete Überzeugung, eine weitere Suche sei nutzlos, weil es eine solche Definition gar nicht geben könne. Eine Position letzterer Art vertritt - generalisiert auf die Begriffe 'Wort', 'Satz' und 'Text' - z.B. R. Rath (1993: 459): "Ich unterstelle also, daß Linguisten zentrale Begriffe (!) ihres Fachs nicht allgemeingültig definieren (kömmen)".

Die Diskussion über die Problematik des Satzkonzepts hat sich in jüngster Zeit noch zugespitzt durch kritische Aussagen über den Status von Sätzen in gesprochener Sprache. Fiehler (1992) zitiert eine Äußerung von Henne: "Geschriebene Sprache geht in Sätzen auf, gesprochene Sprache nicht', Und Rath (1985: 1653) formuliert sogar: "In der gesprochenen Sprache ist der syntaktisch orientierte Begriff des Satzes unbrauchbar". Dementsprechend argumentiert Rath seit langem dafür, bei der Analyse gesprochener Sprache von einer anderen Äußerungseinheit auszugehen (vgl. 1976, 1979, 1992). Schließlich vertritt Fiehler (1992) die noch extremere Position, die Verwendung produktorientierter Äußerungseinheiten sei für die Analyse gesprochener Sprache überhaupt unangemessen und an ihre Stelle müßten prozeßorientierte Kategorien treten.

Wenn in einer Wissenschaft bei der Untersuchung eines bestimmten Phänomens bestimmte scheinbar unüberwindliche Schwierigkeiten auftreten, dann liegt es oft nahe und ist für die emotionale Befindlichkeit angenehm zu fordern, es müsse ein neuer Weg zur Lösung der Probleme gefunden werden. Was allerdings die Einschätzung des Satzkonzepts angeht, so muß man vermeiden, das Kind mit dem Bade auszuschütten. Deshalb lohnt es sich, nochmals folgendes Prüfverfahren durchzuführen.

- Es ist zu untersuchen, inwieweit auch Kommunikation in gesprochener Sprache in Sätzen organisiert ist.

- Sofern neben der Satzorganisation andere Gliederungsverfahren in gesprochener Sprache relevant sind, muß ihr Verhältnis zur Satzeinheit geklärt werden.

- Die verschiedenen Arten von Problemfällen, bei denen eine Satzsegmentierung von Äußerungsketten als nicht möglich erscheint, sind systematisch zu untersuchen und in ihren Konsequenzen für die Frage der Einheitenwahl auszuwerten.

- Unabhängig davon, ob sich der Satz als eine universelle Einheit für gesprochene und geschriebene Sprache herausstellt oder nicht, muß der theoretische und empirische Status dieser Einheit geklärt und eine Satzdefinition angegeben werden.

Im vorliegenden Aufsatz werde ich wesentliche Aspekte dieser vier Schritte bearbeiten und nachweisen, daß der Satz auch die zentrale Äußerungseinheit in der gesprochenen Sprache ist. Hierzu muß das übliche, normativ geprägte Verständnis des Satzbegriffs allerdings durch eine empirisch adäquatere Interpretation abgelöst werden. 


\section{Sätze als relevante Einheiten von Kommunikationsgliederung}

Gegen die Unbrauchbarkeitsannahme von Rath (1985) setze ich provokativ die Gegenthese:

Sprachlich korrekte Äußerungsketten (Texte) - sei es in geschriebener oder in gesprochener Sprache - sind stets vollständig in Sätze zerlegbar.

Schon weil sie ein Allsatz ist, kann ich diese These nicht beweisen, sondern nur exemplarisch begründen und einige Falisifikationsversuche widerlegen. Hierzu will ich ein Beispiel diskutieren, bei dem Rath (1993) dafür argumentiert, daß statt einer Satzsegmentierung eine Unterteilung in andere Äußerungseinheiten anzusetzen sei. ${ }^{2}$ $\mathrm{Zu}$ Recht weist Rath auf die seit Gülich (1970) bekannte Beobachtung hin, daß Kommunikationsteilnehmer in der mündlichen Kommunukation sogenannte Gliederungssignale zur Strukturierung von Texten benutzen und daß somit Äußerungsteile, die zwischen zwei solchen Signalen stehen, evtl. relevante Äußerungeseinheiten bilden. Weiter schreibt Rath: "Dabei wirken lexikalisch-syntaktische Signale mit prosodischen (einschließlich Pausen) zusammen. Wie dieses Zusammenspiel im einzelnen funktioniert, ist bisher noch nicht geklärt. Ungeklärt ist auch die Gliederung von Sprecherbeiträgen ohne explizite Gliederungssignale - also eine schwächere Form der Gliederung, die sich nur auf Intonation und/oder Pausen stützt' (1993: 460). Als eine mögliche Ursache dafür, daß die durch Gliederungssignale gebildeten Einheiten inkompatibel mit Satzeinheiten sein könnten, verweist Rath auf eine von Betten (1976: 225) postulierte Regel: "Sobald du merkst, daß der Hörer verstanden hat, was du ihm mitteilen willst, ist eine Fortsetzung deiner Rede in vielen Situationen unnötig oder unangemessen (Folgen z.B. Satzabbruch oder Ellipse)",

Vor dem so skizzierten theoretischen Hintergrund wollen wir einen Ausschnitt aus dem Gespräch 'Der Intellektuelle' im Korpus Texte gesprochener deutscher Standardsprache (1971: 239f.) betrachten.

1 A du mißverstehst mich *

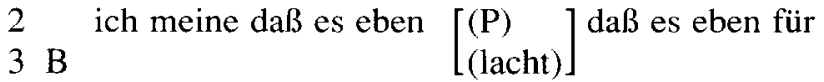

4 A solche Momente eben wichtig ist daß eben nicht a

5 allein auf solcher Basis die Ehe gegründet ist, *

6 B [na gut aber

7 A [daß auch auch gewisse äh gewisse äh geistigen

8 Interessen *

9 B ja:

10 A notwendig sind um dann in der Krise die Ehe zu

2 Wissenschaft lebt zu einem guten Teil auch von kontroversen Diskussionen. Sie sind immer dann konstruktiv, wenn die Diskussionspartner ihre jeweiligen Argumente explizit und präzise formulieren sowie wechselseitig aufeinander eingehen. In diesem Sinne danke ich Rainer Rath ebenso wie meinem Kollegen Reinhard Fiehler für ihren guten argumentativen Gegenpart. 
11 überbrücken, $\left[\begin{array}{l}\text { um die } \\ \text { aber }\end{array}\right]$ Krise überhaupt die

13 A Krisen $\left[\begin{array}{l}\text { zeit zu überbrücken, } \\ \text { oh nein also }\end{array}\right] \begin{aligned} & * \\ & \text { ich }\end{aligned}$

15

bin immer so allergisch dagegen wenn wir auf dem

Intellektuellen und und der geistigen Überlegen-

17 heit da herumpochen und meinen damit weiß was

18 erlangt zu haben, *

19 also ich glaube daß einfache Leute sich auch ihre

20 Worte finden um sich auszusprechen und das sind

21 überhaupt nicht intellektuell zu sein braucht, *

22 ich ich hab mich mit Herrn Frühbold so heute

23 wunderbar über menschliche Probleme unterhalten *

24 und der wird nicht als Intellektueller

25 bezeichnet *

26 also ich weiß nicht äh dagegen muß ich mich

27 unheimlich wenden, weißt du *

28 das ist so die Art die wir Studenten einnehmen ,

29 nicht, *

30 der Intellektuelle, nicht, *

31 der Einblick hat $(\mathrm{P})$ weißt du so *

32 zu wissen was die Welt im Innersten zusammenhält

33 A $\left[\begin{array}{l}\mathrm{ja} \\ \text { nein nein }\end{array}\right] \stackrel{\text { so mein ich das gar nicht }}{*}$

In dem Transkriptionsausschnitt werden folgende Notationen verwendet. A und B dienen als Sprechersiglen, mit [ ] wird simultanes Sprechen markiert, Komma und Hochkomma zeigen fallende bzw. steigende Intonationskontur an, der Doppelpunkt markiert Lautdehnungen, und mit $(P)$ werden Pausen notiert. Außerdem zeigt das Zeichen * jeweils das Ende der nach der Analyse von Rath anzunehmenden dreizehn Äußerungseinheiten an.

Bei acht der dreizehn aufgrund vorkommender Gliederungssignale postulierten Äußerungseinheiten fällt die Grenzziehung mit der intuitiven Unterteilung in Sätze zusammen, und zwar gilt dies für die Grenzziehung in den Zeilen 1, 13, 18, 21, 23, 25, 27 und 33. Insofern kann die Satzsegmentierung für die Gliederung des Gesprächsausschnitts nicht generell irrelevant sein. In einem Fall (Z. 21) läßt sich die Grenzziehung sogar nur bei Berücksichtigung der Satzsegmentierung eindeutig begründen; denn außer der fallenden Intonation, die allein kein ausreichendes Gliederungssignal bildet (vgl. Z. 27, 28, 30), gibt es kein weiteres formales Gliederungssignal.

Weiterhin müssen wir prüfen, wie die restlichen fünf Fälle einzuschätzen sind, bei denen die Gliederung von Rath nicht mit der Satzsegmentierung übereinstimmt. Die beiden Grenzziehungen am Ende von Zeile 5 und 8 bilden nur eine interne Unterteilung des von Zeile 2 bis Zeile 13 reichenden Satzes und stellen somit die gröbere Satzsegmentierung nicht in Frage. Welchen Status haben sie dann? Für die Grenz- 
ziehung nach Zeile 5 gibt Rath keine Begründung an. Man kann aber davon ausgehen, daß er die Verhältnisse an dieser Stelle ähnlich einschätzt wie bei der Grenzziehung am Ende von Zeile 8. Insofern brauchen wir uns nur mit Raths Argumentation an letzterer Stelle auseinanderzusetzen. Richtig ist, daß B mit dem Rezeptionssignal ja: in Zeile 9 den Redefluß von A kurzzeitig stoppt und eine relevante Äußerungseinheit zu konstituieren versucht. Auf die von Rath genannten Ursachen für den Unterbrechungsversuch von B brauchen wir nicht einzugehen. Die entscheidende Frage ist aber, ob Signale wie na gut und ja ausreichen, um Äußerungseinheiten abzuschließen. Die Antwort auf diese Frage heißt: dies gilt nicht generell und insbesondere nicht in den beiden diskutierten Fällen. Die formale Strukturierung von mündlicher Kommunikation ist nämlich eine interaktive Aufgabe aller Beteiligten. Konkret bedeutet dies: Ein Strukturierungsversuch eines der Teilnehmer tritt nur dann in Kraft, wenn er von dem/den anderen explizit ratifiziert oder zumindest stillschweigend geduldet wird. Wenn A in dem Gesprächsausschnitt also nach Zeile 5 bzw. Zeile 8 seine Äußerung abgebrochen und das Rederecht an B übergeben oder selbst eine neue Äußerung begonnen hätte, dann könnte seine abgebrochene Äußerung evtl. als interaktiv abgeschlossene Einheit gelten. Diese Bedingung ist aber in beiden Fällen nicht erfüllt, weil A seine Äußerung syntaktisch in Zeile 7 bzw. Zeile 10 fortführt. Somit sind die beiden Grenzziehungen am Ende von Zeile 5 bzw. Zeile 8 nicht berechtigt. Das impliziert jedoch nicht, daß die beiden Signale na gut und $j a$ an den betreffenden Stellen funktionslos werden. Sie behalten nämlich ihre Funktion als Rezeptionssignale. Diese Funktion besteht unabhängig von Aufgaben der Textgliederung darin, daß nach inhaltlich relevanten Äußerungsabschnitten Zustimmung oder Ablehnung, Verständnis oder Unverständnis u.ä. manifestiert wird. Der Spielraum für die Produktion von Rezeptionssignalen ist sehr groß: einerseits sind sie grundsätzlich optional, andererseits können sie an nahezu jeder syntaktischen Position vorkommen. Aus diesem Grund werden durch sie keine eigenständigen Äußerungseinheiten definiert, und sie besitzen nur in Kombination mit anderen Strukturierungsmitteln äußerungsgliedernde Kraft.

Was nun die Grenzziehungen in Zeile 29, 30, 31 anbetrifft, so sind zwei Analysemöglichkeiten zu diskutieren. Entweder bildet Zeile 28-29 einen Satz, und Zeile 30-31 sowie Zeile 32-33 sind elliptische Äußerungen; die Behandlung von Ellipsen will ich auf Abschnitt 4 verschieben. Oder aber die gesamte Passage von Zeile 28-33 bildet einen Satz, wobei Zeile 30-31 sowie Zeile 32-33 als Nachträge in einer Art 'Doppelpunkl'-Konstruktion an Zeile 28-29 angeschlossen sind. Bei dieser zweiten Analysemöglichkeit bilden die betreffenden Grenzziehungen eine interne Untergliederung des Gesamtsatzes und widersprechen insofern nicht der Satzsegmentierung. An dieser Untergliederung sind zwei Punkte interessant. Erstens korrespondiert sie wieder mit syntaktisch relevanten Einheiten. Die Grenze nach Zeile 28 schließt gerade einen Teilsatz $a b$, der anschließend in drei Informationsportionen expandiert wird. Diese Portionen sind als Phrasen oder Nebensätze formuliert, und nach jeder Portion könnte der Gesamtsatz beendet werden. Zweitens fällt auf, daß in Zeile 29 und 30 die Negation nicht, die zusammen mit der steigenden Intonationskontur die Funktion einer Zustimmungsaufforderung (tag-Partikel) hat, bei A nicht die gewünschte Reaktion zeigt. Der Grund hierfür liegt vermutlich darin, daß A der 
inhaltlichen Position von B nicht zustimmen wollte. Zugleich verhindert die fehlende Reaktion offensichtlich einen Abschluß des begonnenen Satzes.

Die Ergebnisse unserer Analyse legen die Vermutung nahe, daß die formale Unterteilung durch Gliederungssignale und die syntaktische Segmentierung weder konträr noch voneinander unabhängig sind. Vielmehr tragen offensichtlich beide Verfahren gemeinsam/synergetisch zur Text-/Kommunikationsgliederung bei. Wie dieses Zusammenwirken im einzelnen funktioniert, muß noch genauer untersucht werden. Ein für die Relevanzeinschätzung wichtiger Sachverhalt ist aber sofort erkennbar. Die syntaktische Gliederung ist im mikrostrukturellen Bereich differenzierter und zugleich stabiler als die Segmentierung durch Gliederungssignale. Einerseits wird nämlich nicht jede Phrase/Konstituente im Satz oder jeder Satz durch Gliederungssignale abgegrenzt, zum anderen können vorhandene und speziell lexikalische Gliederungssignale evtl. getilgt oder an bisher nicht abgegrenzten Stellen neue Signale eingefügt werden, ohne daß sich der Bedeutungsaufbau der betreffenden Äußerungen und die zugrundeliegende syntaktische Gliederung wesentlich ändern. Beispielsweise lassen sich im obigen Gesprächsausschnitt ohne wesentlichen Funktionsverlust die satzumschließenden lexikalischen Gliederungssignale von Zeile 26 und 27 entfernen, und dasselbe gilt für die satzinternen Signale zwischen Zeile 28 und 32. Umgekehrt ist im makrostrukturellen Bereich eine Gruppierung von Sätzen zu größeren Äußerungsabschnitten nur mit Hilfe von Gliederungssignalen (unter Einrechnung von Konjunktionen) möglich, und hier sind sie als formales Gliederungsverfahren konkurrenzlos.

Insgesamt gesehen scheint es sich also zu empfehlen, die Frage nach den Mitteln von Kommunikationsgliederung nicht eindimensional zu beantworten, sondern von einem komplexen dynamischen Strukturierungssystem auszugehen. Eine genügend allgemeine theoretische Basis für ein solches System soll im folgenden Abschnitt skizziert werden.

\section{Gliederungsverfahren in der Kommunikation und Stellenwert der Satz- gliederung}

Die Durchführung jeder Art von Kommunikation wird durch die Bewältigung bestimmter genereller Aufgaben der Verständigungssicherung überlagert. Wie in Untersuchungen zum sogenannten Hamburger Verständlichkeitskonzept bereits vor längerer Zeit nachgewiesen wurde (vgl. Langer et al., 1974), bildet Gliederung einen zentralen Faktor von Textverständlichkeit. Die für diesen Faktor erforderliche Operationalisierung läßt sich auf den verschiedenen linguistischen Ebenen durch einschlägige Gliederungsverfahren präzisieren. Grundsätzlich ist zwischen Kontinuitäts- und Diskontinuitätsverfahren zu unterscheiden. Mit einer sprachlichen Markierung von Diskontinuität wird eine Grenze zwischen zwei aufeinanderfolgenden Kommunikationsteilen gezogen. Für eine solche Grenzziehung kann es eigene, nur für diese Aufgabe zuständige Signale geben, wie z.B. bestimmte Gliederungssignale. Die Grenzmarkierung wird aber auch oft durch konventionalisierte Restriktionen bei der Verwendung anderer Einheiten vollzogen; beispielsweise signalisiert 
das Vorkommen eines Artikels i.a. gleichzeitig den Beginn einer Nominalphrase. Theoretisch kann man zwischen Anfangs-, End- und Mittemarkierungen unterscheiden. Bei den Gliederungssignalen ist z.B. also ein typisches Anfangs-, die Pause ein Mitte- und $j a$ ein Endsignal. Die Stabilität solcher Einstufungen ist allerdings sowohl sprachhistorisch wie aktualgenetisch nicht sehr groß. So stellt nämlich also primär ein mitteanzeigendes Verknüpfungssymbol der Folgerung dar, und ja wird speziell in Kombination mit anderen Gliederungssignalen auch zur Anfangsmarkierung verwendet (vgl. Kindt, 1993a). Ursache für diese Instabilität ist, daß durch die Kombination unterschiedlicher Gliederungsmittel die lineare Entfernung eines Gliederungssignals zum Ende der vorausgehenden und Anfang der folgenden Äußerung verändert werden kann. Beispielsweise führt eine lange Pause nach der Formulierung von ja dazu, daß man dieses Signal als in der Nähe der Vorgängeräußerung liegend wahmimmt, während eine vorherige lange Pause das Signal in die Nähe der Nachfolgeäußerung rückt. Insofern ist es manchmal schwierig, Gliederungssignale eindeutig entweder der Vorgänger- oder der Nachfolgeräußerung zuzuordnen (vgl. Z. 26, 27 und 31 im obigen Gesprächsausschnitt). Eine eindeutige Entscheidung ist aber nicht in jedem Fall erforderlich, denn es kann sich auch um eine Mittemarkierung bzw. eine Pivot-Konstruktion handeln, die nach beiden Seiten hin orientiert ist.

Im Gegensatz zur Trennungsfunktion von Diskontinuitätsverfahren wird mit Hilfe von Kontinuitätsverfahren deutlich gemacht, welche Teile einer Kommunikation als zusammengehörig betrachtet werden sollen. Eine solche Zusammengehörigkeit kann explizit durch prosodische Markierungen oder lexikalische Verknüpfungssymbole wie etwa Konjunktionen signalisiert werden. Ein anderes, stärker implizites Verfałlren der Kontinuitätsmarkierung basiert demgegenüber auf der Realisierung syntagmatischer Abhängigkeitsbeziehungen durch Kookkurrenzrestriktionen oder Kongruenzbedingungen (wie bei Subjekt und finitem Verb). Außerdem liegt eine Einstufung zweier Kommunikationsteile als zusammengehörig nahe, wenn sie ohne dazwischenliegenden Grenzsignal unmittelbar benachbart sind (und nicht ohne Funktionsverlust verschoben werden können) oder wenn sie (wie bei Überbrückungsreparaturen; vgl. Abschnitt 4) durch partielle Wiederholung von Äußerungsteilen aneinander anknüpfen.

Die Gliederung von Kommunikation bedient sich sowohl der Kontinuitäts- als auch der Diskontinuitätsverfahren, und deshalb kann man zunächst fragen, wo ihre jeweiligen Stärken und Schwächen liegen. Grundsätzlich - so ist leicht einzusehen benötigt man für die Bildung größerer kommunikativer Einheiten eher manifeste, eigenständige Grenz- und Verknüpfungssignale als bei kleineren Einheiten, um die linear größeren Entfernungen mental leichter überbrücken zu können. Daneben gibt es eine Abhängigkeit von der Informationsebene: Beispielsweise sind semantische im Gegensatz zu syntaktischen Abhängigkeitsbeziehungen aufgrund der menschlichen Gedächtnisorganisation auch bei größeren linearen Entfernungen noch identifizierbar. Deshalb ist eine Einheitenbildung über Pronominakoreferenz auch satzübergreifend möglich. Schließlich scheinen aber Kontinuitätsmarkierungen bei der Bildung kleinerer und Diskontinuitätsmarkierungen bei der Bildung größerer Einheiten jeweils dominanter zu sein. 
Insgesamt gesehen muß man von einer komplexen wechselseitigen Abhängigkeit und Interaktion beider Verfahrenstypen ausgehen. So wird einerseits durch das Ziehen zweier Grenzen, also durch die Abtrennung dreier Kommunikationsteile, der in der Mitte liegende Teil i.a. als zusammengehörige Einheit ausgezeichnet. Umgekehrt grenzt eine Kontinuitätsmarkierung gleichzeitig die beiden zu verbindenden Teile auf hierarchisch niedrigerer Stufe gegeneinander ab; dies erklärt, warum Verknüpfungssymbole wie also und und auch die Funktion von Gliederungssignalen haben können. Außerdem liegt es nahe, daß zwei nicht unmittelbar benachbarte Kommunikationsteile, die als zusammengehörig identifiziert sind, auch den durch sie abgegrenzten Mittelteil in eine gemeinsame Einheit aufnehmen; dieser Fall liegt z.B. bei dem sogenannten Phänomen der Satzklammer vor, bei der das finite Verb und ein diskontinuierlich am Satzende liegender Verbalkomplex die inneren Satzglieder zu einer Einheit (etwa einer Verbalphrase) unuschließen (vgl. Kindt, 1993c).

Wenn zwei Kommunikationsteile durch ein Grenzsignal voneinander getrennt sind, dann kann ihre Ausdehnung nicht immer durch Übergang zu den nächsten, weiter außen liegenden Grenzen ermittelt werden, weil diese möglicherweise zu einer anderen Art der Untergliederung gehören. In einem solchen Fall läßt sich die jeweilige Ausdehnung evtl. durch Zusammengehörigkeitsbeziehungen einer speziellen Informationsebene identifizieren. Umgekehrt reicht für die Bestimmung von Einheiten die Analyse von Zusammengehörigkeitsbeziehungen oft nicht aus, sondern man wertet sie in bezug auf schon gefundene Grenzen aus. In solchen Fällen ergänzen sich also beide Verfahren. Dies gilt in spezieller Weise für hierarchische Gliederungen.

Grundsätzlich ergibt sich die Notwendigkeit hierarchischer Gliederungen aus dem Verständlichkeitsfaktor 'Einfachheit', weil Strukturen mit wenigen Untergliederungen auf jeder Hierarchiestufe mental leichter $\mathrm{zu}$ verarbeiten sind als einstufige/ 'flache' Untergliederungen mit hoher Zahl von Elementen. Wir wollen zwei Arten von Hierarchieerzeugung kurz ansprechen. Erstens ist denkbar, daß die Grenzsignale zweier Informationsebenen an bestimmten Stellen zusammentreffen. Dieser Fall wurde unlängst in einem DFG-Projekt von mir am Beispiel von Erzählungen genauer untersucht (vgl. Kindt, 1993a). Wenn nämlich Signale wie z.B. ja, und und dann kombiniert werden, dann faßt man sie aufgrund ihrer Benachbartheit als zusammengehörig auf, und sie bilden im Vergleich zum Vorkommen eines einzelnen Signals eine 'starke' Grenzmarkierung. Der Stärkegrad von Grenzsignalen konstituiert dann eine Zusammengehörigkeitsbeziehung unter ihnen, d.h. die Ermittlung einer Einheit auf einer bestimmten Hierarchiestufe kann durch Übergang von einem Signal zu dem nächsten Signal des gleichen Stärkegrades geschehen. Zweitens entsteht eine Hierarchisierung evtl. dadurch, daß von zwei zusammengehörigen Kommunikationsteilen der eine relativ selbständig und der andere als Informationsergänzung des ersten unselbständig ist. Klassisches Beispiel für so begründete Hierachien sind die Dependenzstrukturen der Valenzgrammatik (wobei die Richtung der postulierten Abhängigkeitsbeziehungen wie etwa im Fall der Subjekt-Verb-Beziehung teilweise inkorrekt angegeben wird; vgl. Kindt, 1993c).

Nach diesen sehr generellen theoretischen Überlegungen über Gliederungsverfahren in der Kommunikation möchte ich jetzt konkreter auf Verfahren und Stellenwert 
der Satzgliederung eingehen. Nach den Ergebnissen des erwähnten Projekts bilden parataktisch, und zwar syndetisch oder asyndetisch aneinander angeschlossene Sätze die Basiseinheiten für den Aufbau von Makrostrukturen in der natürlichsprachigen Kommunikation. Für die Aufgabe der formalen Gliederung verwenden Produzenten in der gesprochenen Sprache jeweils Kombinationen von Anfangsgliederungssignalen zur Abgrenzung größerer Einheiten, nämlich der sogenannten Äußerungs- oder Kommunikationsphasen. Obschon es ein einheitliches Repertoire solcher Anfangssignale gibt, ist die Auswahl und Kombination solcher Signale teilweise sprecherspezifisch. Das Prinzip der Phasenbildung wird im oben diskutierten Gesprächsausschnitt auch an dem Redebeitrag von B deutlich. B benutzt zur Phasenmarkierung eine Kombination von Signalen, in der stets das Anfangssignal also und eine epistemische Formel wie z.B. ich weiß nicht vorkommen. Danach ist der Redebeitrag von B zwischen Zeile 14 und 33 in drei Äußerungsphasen zu unterteilen, wobei die erste Phase nur aus einem Satz besteht und die zweite und dritte Phase jeweils zwei asyndetisch aneinander angeschlossene Sätze enthält.

Die Phasenbildung wird zwar i.a. kohärent zur inhaltlichen Gliederung von Makrostrukturen gestaltet, diese beiden Gliederungen fallen aber nicht immer zusammen. Beispielsweise kann man in Erzählungen feststellen, daß die Vorgeschichte des darzustellenden Ereignisses, die inhaltlich gesehen einen eigenen Abschnitt bildet, entweder in der Phase der einleitenden Orientierung oder im Zusammenhang mit dem zentralen Ereignis/der Komplikation erzählt wird. In unserem Gesprächsausschnitt entspricht die Phasierung des Redebeitrags von B aber genau der inhaltlichen Unterteilung in drei Gegenargumente zu der vorausgehenden These von $\mathrm{A}$.

Die Identifizierung von Sätzen als den Basiseinheiten von Phasen geschieht im wesentlichen durch die Erkennung syntaktischer Zusammengehörigkeitsbeziehungen. In einfachen Sätzen etwa wird die Zugehörigkeit einer Phrase zu einem Satz weitgehend durch die Prüfung entschieden, ob die Phrase valenzmäßig zum finiten Verb oder zu anderen benachbarten Phrasen paßt; wenn dies nicht der Fall ist, muß die Phrase zu einem neuen Satz gehören. Zugleich ergeben sich durch die für Satzglieder geltenden Positionsrestriktionen spezifische Möglichkeiten der Identifizierung von Satzanfang oder -ende. Im allgemeinen reicht das so skizzicrte Verfahren für eine eindeutige Satzsegmentierung aus. Trotzdem werden auch Markierungen anderer Informationsebenen für die Segmentierung ausgewertet und in mehrdeutigen Fällen zur Entscheidung benutzt. Dies gilt insbesondere für Interpunktionen in der geschriebenen bzw. für Gliederungssignale und Prosodie in der gesprochenen Sprache. Beispielsweise läßt sich die Äußerung Karl arbeitet heute ist er guter Laune nur bei Vorliegen zusätzlicher Strukturierungsmerkmale eindeutig in zwei Sätze unterteilen. Genereller sind bestimmte satzbegleitende Strukturierungen der genannten Ehenen konventionell erwarthar, und deshalb werden Sätze ohne entsprechende Markierungen als sprachlich inakzeptabel bzw. schwer verstehbar eingeschätzt.

Die Phasenbildung mit Hilfe von Anfangssignalen ist in starkem Maße von den Bedingungen und Zielen der Äußerungsproduktion her bestimmt. Demgegenüber fällt im obigen Gesprächsausschnitt auf, daß die vorkommenden sprecherseitigen 
Gliederungsendsignale rezipientenorientiert formuliert werden (vgl. etwa also ich weiß nicht äh am Satzanfang von Z. 26 vs. weißst du am Satzende von Z. 27). Zugleich liegen diese Signale (inklusive der fallenden Intonationskontur) stets an Stellen, bei denen der bisher produzierte Satz potentiell beendet werden kann, aber faktisch nicht immer beendet wird. Hieraus ergibt sich, daß die evtl. mit Rezeptionssignalen kombinierten Gliederungsendsignale eine andere Funktion als Phasensignale haben. Auch sie sind in dem diskutierten Gesprächsausschnitt mit der Satzproduktion kompatibel, aber nur im potentiellen Sinne. Ob an den durch sie bestimmten Grenzen ein Satz und/oder eine Phase beendet wird, liegt nicht von vornherein fest, sondern wird erst interaktiv in Abhängigkeit von den jeweiligen Formulierungsinteressen der Beteiligten entschieden. Deshalb muß im Endsignal auch keine zugehörige Hierarchiestufe durch die Wahl einer speziellen Signalkombination manifestiert werden, sondern dies kann im nachfolgenden Anfangssignal geschehen. Neben der syntaktischen Organisation und der Regelung des Rederechts haben die Endsignale auch eine wesentliche Funktion auf der Ebene der Verständigungssicherung bezüglich Äußerungsinterpretation und Zustimmung oder Ablehnung von Sachverhalten. Wenn dabei wie an den schon diskutierten Stellen Zeile 29 und 30 unseres Beispiels ein vom Rezipienten angefordertes Ratifikationssignal ausbleibt und auch keine Zurückweisung erfolgt, dann wird damit indirekt auch der Übergang zu einer neuen Gesprächseinheit abgelehnt.

Im Anschluß an letztere Überlegungen muß noch systematischer diskutiert werden, welchen Stellenwert Gliederungssignale für die Konstitution von Äußerungseinheiten unterhalb der Satzebene besitzen. Im Zusammenhang mit der Beispielanalyse in Abschnitt 2 haben wir schon gesehen, daß die innerhalb von Säzen plazierten Rezeptionssignale keine eigenständigen Satzuntergliederungen definieren und somit keine Äußerungseinheiten konstituieren. Dasselbe gilt für satzintern eingeschobene Produktionssignale wie z.B. das Hesitationssignal $e h$, weil deren Äußerung durch Zeitprobleme im Planungs- und Formulierungsprozeß bedingt ist. Die Formulierung von Rezeptions- und Produktionssignalen bildet - genauer gesehen - nur einen Spezialfall der Durchführung von satzinternen Reparaturen. Auch solche Reparaturen konstituieren keine Äußerungseinheiten, die unabhängig von der übrigen syntaktischen Organisation in Sätzen sind. Um dies nachzuweisen muß man allerdings zeigen, von welchen grammatischen Konstruktionen Reparaturen Gebrauch machen (vgl. Abschnitt 4). Aufgrund bestimmter Positionspräferenzen und -restriktionen kann die syntaktische Gliederung von Sätzen auch durch das Vorkommen von Reparaturen interaktiv unterstützt werden. Dies erklärt die Sonderstellung der Satzendebzw. Nachfeldposition einerseits für Aktivitäten der Verständigungssicherung (wie in Zeile 27, 29, 30, 31, 33 unseres Beispiels) und andererseits für die Produktion von nachgeschobenen Informationsportionen in Form von Ausklammerungskonstruktionen und Nachträgen.

Abschließend will ich noch einige Bemerkungen zu der Frage machen, warum die Einheit des Satzes so zentral für den Aufbau von Kommunikation ist. Zunächst ist klar, daß man überhaupt eine stabile Organisationseinheit für die Darstellung elementarer Sachverhalte braucht. In einer solchen Darstellung wird jeweils über einen gewählten Gegenstand (Thema) eine bestimmte Aussage durch Angabe einer Prädi- 
kation (Rhema) gemacht. Sätze sind ein kommunikatives Mittel für derartige Darstellungen, und ihnen liegt ein sozial sehr stabiles Organisationsschema für Prädikationen zu einzelnen relevanten Informationsaspekten zugrunde. Dabei fällt die Thema-Rhema-Gliederung allerdings nicht zwangsläufig mit der klassischen Subjekt-Prädikat-Unterscheidung zusammen, sondern die verschiedenen Informationsaspekte können je nach Vorinformationen unterschiedlich gruppiert werden. Zugleich lassen sich im Rahmen von Sätzen elementare Sachverhaltspräsentationen mit Hilfe eines begrenzten Repertoires an Verknüpfungsformen zu komplexen Darstellungen kombinieren. Die Verstehbarkeit komplexer Sätze ist allerdings stark eingeschränkt, und deshalb wird von den potentiellen Verknüpfungsmöglichkeiten faktisch nur in geringem Ausmaß Gebrauch gemacht, bzw. statt dessen die Organisationsform der Phasenbildung bevorzugt. Sätze erfüllen also einerseits die Minimalitätsbedingung hinsichtlich der Darstellung elementarer Sachverhalte. Andererseits bietet ihre konstruktionsmäßige Begrenzung verständigungsökonomische Vorteile. Für letzteren Aspekt spielen insbesondere zwei informationsdynamische Faktoren eine wesentliche Rolle. Erstens muß es Aufgabe einer kommunikativ effektiven Sachverhaltsdarstellung sein, im Rahmen eines möglichst einfachen Organisationsschemas ein Höchstmaß an semantischer Flexibilität zu erreichen. Zu diesem Zweck sind Sätze so strukturiert, daß während ihrer sukzessiven Verarbeitung noch bestimmte Entscheidungen über syntaktische und semantische Zuordnungen offenbleiben. Deshalb muß es einen Zeitpunkt geben, an dem spätestens derartige Entscheidungen endgültig getroffen werden, und dieser Zeitpunkt darf aus Gründen der beschränkten Gedächtniskapazität bzw. -organisation nicht zu weit hinausgeschoben werden. Zweitens nehmen Kommunikationsteilnehmer während der Sprachverarbeitung auch schon bestimmte naheliegende syntaktische und/oder semantische Zuordnungen vor, die später evtl. aufgrund neuer Informationen revidiert werden müssen. Dies ist das sogenannte Nichtmonotoniephänomen von natürlichsprachiger Informationsverarbeitung, das sich auf syntaktischer Ebene gut an Garden-Path-Sätzen wie Alkohol entfernt mit einem Lappen hinterläßt keine Flecken demonstrieren läßt (bei sukzessiver Rezeption wird entfernt zunächst als finites Verb und später als Partizip analysiert; vgl. Kindt, 1991, 1993b). Auch der Zeitpunkt, bis zu dem mögliche Zuordnungsrevisionen zugelassen sind, darf zweckmäßigerweise nicht zu weit hinausgeschoben werden. Tatsächlich bildet das Ende von Sätzen die Stelle, an der relativ viel Verarbeitungszeit benötigt wird, um offengebliebene Zuordnungen, also z.B. eine Thema-Rhema-Untergliederung, durchzuführen und notwendige Revisionen vorzunehmen. Eine spätere Revision durch die Information nachfolgender Sätze ist zumindest für die syntaktische Ebene kaum möglich. Beispielsweise würde in Die Mutter beschimpft das Kind. Sie hat vergessen, die Hausaufgaben nachzusehen aus semantischen Gründen eine rückwirkend geänderte Analyse von das Kind als Subjekt und von die Mutter als Objekt des ersten Satzes naheliegen; dieser Effekt wird aber nicht erreicht. Insofern bilden Sätze speziell auf syntaktischer Ebene eine maximale, autonome Informationseinheit, und diese Charakterisierung kommt der aus einem anderem Argumentationszusammenhang stammenden Satzdefinition von Bloomfield (1926) sehr nahe, auf die wir gleich eingehen wollen. 


\section{Ellipsen und Reparaturen als Testfälle für die Satzsegmentierung}

Mittlerweile haben wir zwar durch Beispielanalyse und theoretische Diskussion die These plausibel gemacht, daß auch Texte der mündlichen Kommunikation in Sätze zerlegbar sind. Nach wie vor gibt es aber bestimmte Typen möglicher Gegenbeispiele gegen die These. Hierzu gehören einerseits Ellipsen und andererseits Äußerungen mit Satzabbriichen oder Reparaturen. Diese Konstruktionen müssen wir jetzt untersuchen. Das Resultat dieser Untersuchung kann nur heißen: Entweder ist die These geeignet zu modifizieren, oder es muß nachgewiesen werden, daß bei derartigen Daten entgegen dem ersten Anschein doch Sätze vorliegen. Es ist klar, daß eine solche Untersuchung nur erfolgreich sein wird, wenn man über eine Satzdefinition verfügt. Aus Gründen, die in Abschnitt 5 genauer dargestellt werden, ist es zweckmäßig, eine primär grammatikunabhängige Satzdefinition zu suchen. Insofern wäre es ungünstig, wenn man als Definitionsbedingung z.B. den Sachverhalt verwenden würde, daß einfache Sätze (im Normalfall) aus einer Subjektnominalphrase und einer Verbalphrase bestehen; denn die Formulierung dieses Sachverhalts setzt schon grammatische Kategorien voraus, die selbst in spezifischem Zusammenhang mit dem Satzkonzept stehen. Demgegenüber läßt die Satzdefinition von Bloomfield (1926) eine grammatikunabhängige Interpretation zu. Nach dieser Definition ${ }^{3}$ ist ein Satz eine maximale Form von Äußerungen. Diese Formulierung wird aufgrund anderer, vorhergehender Definitionen von Bloomfield paraphrasiert durch Ein Satz ist eine Form, die in einer gegebenen Äußerung nicht Teil einer größeren Konstruktion ist. Auch in dieser Version hat die Definition von Bloomfield noch einen wesentlichen Nachteil. Selbst bei Berücksichtigung des Kontextes der Definitionsformulierung weiß man nämlich nicht, mit welchen Verfahren man das Kriterium der Eingebettetheit in Konstruktionen überprüfen soll. M.a.W.: Es fehlt eine empirische Operationalisierung der Definition, mit deren Hilfe man für vorgegebene Äußerungen entscheiden kann, ob sie das Kriterium erfüllen oder nicht. Obwohl im strukturalistischen Forschungsparadigma die Notwendigkeit einer Operationalisierung nicht als zentrales Problem erkannt wurde, hat es weitere Formulierungsversuche in Richtung auf eine operationalisierte Satzdefinition gegeben (vgl. z.B. Heringer, 1970: 42). In Erweiterung der Formulierung von Heringer habe ich (1985: 185) folgenden approximativen Definitionsvorschlag gemacht: Der Satz ist die kleinste selbständige sprachliche Einheit, d.h. daß deren Vertreter in Texten frei vorkommen können. Dabei soll die Bedingung des freien Vorkumnens durch das Verfahren der Permutation/Verschiebung der betreffenden Textteile empirisch überprüft werden. Präziser gesagt, bedeutet dies, daß ein Textteil dann ein Satz ist, wenn er von seiner Position aus vor oder hinter jeden Satz in dem Text bzw. auch in anderen Texten geschoben werden kann, ohne daß dadurch die Eigenschaft der sprachlichen Korrektheit der betreffenden Texte verlorengeht. Da zu Beginn der Unterteilung eines

\footnotetext{
Die Originalformulierung in Bloomfields Definition (1926: 158) lautet: A maximum construction in any utterance is a sentence. Thus, a sentence is a construction which, in the given utterance, is not part of any larger construction. Every utterance therefore consists of one or more sentences, and even such utterances as Latin pluit, English Fire! or Ouch! are sentences".
} 
Textes in Sätze noch unbekannt ist, wo Satzgrenzen innerhalb des Textes anzusetzen sind, schwächı nnan das Verschiebbarkeitskeitskriterium so ab, daß nur eine Verschiebung des betreffenden Textteils an den Anfang und an das Ende des Textes gefordert wird. Auch in der abgeschwächten Version sind mit unserem Definitionsvorschlag noch bestimmte Probleme verbunden, auf die ich aber erst in Abschnitt 5 genauer eingehen will. Zunächst soll aber geprüft werden, welche Konsequenzen der Definitionsvorschlag für die Beurteilung der genannten problematischen Konstruktionen hat.

Die übliche Vorgehensweise bei der Satzsegmentierung basiert nicht auf der Anwendung des Verschiebbarkeitskriteriums, sondern orientiert sich am vorverständlichen Satzbegriff oder an den Aussagen von Grammatikmodellen, die ein (mehr oder weniger vollständiges) System von Regeln für die Produktion von Sätzen angeben. Wir wollen uns vorstellen, daß ein schrift- oder gesprochensprachlicher Text auf diese Weise segmentiert worden ist und daß zwischen zwei als Sälzen $S_{1}$ und $\mathrm{S}_{2}$ identifizierten Textteilen ein Äußerungsstück A liegt, das kein Satz zu sein scheint. Wir müssen nun verschiedene Arten von Problemfällen unterscheiden und getrennt behandeln. Grundsätzlich ist jedoch festzustellen, daß solche Problemfälle i.a. nur einen kleinen Teil im Gesamtbereich der Textsegmente ausmachen und daß die unmittelbar als Sätze identifizierbaren Segmente überwiegen. Zugleich betreffen die Problemfälle stets nur syntaktisch sehr rudimentäre Äußerungsteile und reichen bei weitem nicht an die Ausdruckskraft komplexerer Sätze heran. Insofern wäre es ohnehin nicht legitim, aus dem Vorkommen solcher Problemfälle auf die Irrelevanz des Satzkonzepts zu schließen.

Ein erster Typ von Problemfällen liegt vor, wenn das betreffende Äußerungsstück A zwar nicht den üblichen normativen Erwartungen an Sätze genügt, aber frei verschiebbar ist und deshalb im Sinne unserer Definition einen Satz bildet. Genauer sind in Abhängigkeit von der Art der syntaktischen 'Defizite' drei Subfälle zu unterscheiden.

Fall la: A entspricht nicht den Vollständigkeitserwartungen von Sätzen, d.h. bestimmte, im Normalfall realisierte Satzglieder oder Elemente von ihnen fehlen. Prototypisches Beispiel für diesen Fall bilden die sogenannten Telegrammellipsen.

\section{(1) Oma gut angekommen.}

Wie ist das Fehlen der Kopula ist in (1) theoretisch einzuschätzen? Für die Modellierung kann man annehmen, daß die Anwendung bestimmter, für die Erzeugung 'vollständiger' Sätze erforderlicher Regeln (z.B. die lexikalische Einsetzung von ist) unterbleibt, ansonsten aber ein korrekter Erzeugungsprozeß vorliegt. Die Produktion reduzierter/elliptischer Äußerungen ist evtl. dadurch verursacht, daß man sich aus ökonomischen Gründen die Formulierung redundanter/unmittelbar ergänzbarer Äußerungsteile erspart. Ursache für die Wahl elliptischer Formulierungen können aber auch stilistische Effekte sein. Ein vermutlich diesem Funktionstyp zuzurechnendes Beispiel findet man bei Rath (1993: 469).

(2) Und dann werden pro Tach vierzig Ehen geschlossen, $(P)^{*}$ alles kühl und sachlich $^{*}$ 
Außer der Kopula können noch andere, aber nicht beliebige Äußerungselemente ausgelassen werden.

\section{(3) Großer Mann von kleinem Hund gebissen}

Bei Beispielen wie (3) wird die Ergänzung des unbestimmten und nicht des bestimmten Artikels erwartet; zugleich zeigt (3), daß nicht immer ein reines Auslassungsphänomen vorliegt, weil die Notwendigkeit einer starken Flexion bei Fehlen des Artikels evtl. auf das Adjektiv übergeht. Beispiele wie (1)-(3) lassen sich als satzwertige Ellipsen klassifizieren, die sowohl syntaktisch wie semantisch kontextunabhängig sind. Eine semantische Unabhängigkeit liegt bei satzwertigen Ellipsen aber nicht generell vor.

\section{(4) Schmeckt gut,}

In diesem Beispiel ist als Subjekt/Thema das zu ergänzen, wenn man eine minimale semantisch äquivalente Vervollständigung sucht. Wie das zu interpretieren ist, bzw. für welches Referenzobjekt eine Prädikation angegeben wird, hängt in der elliptischen ebenso wie in der vervollständigten Formulierungsversion vom Kontext ab. Grund für die Auslassung des Subjekts ist eine unmittelbare Präsenz des thematisierten Referenzobjekts, und als Modellierungsansatz kann man wieder die Unterdrückung bestimmter Regelanwendungen bei gleichzeitiger direkter Anknüpfung der Prädikation an das Referenzobjekt zugrunde legen.

Subjekt- und Kopulaauslassung werden häufig miteinander kombiniert (vgl. evaluative Einwortwätze wie Toll). Dieser Fall ist auch bei folgendem Beispiel von Rath (1993: 464) gegeben.

\section{(5) Nein, wir haben den Stand schließen lassen nich' * Geschäftsschädigung,*}

Nach Raths Interpretation wird mit dem Äußerungsteil Geschäftsschädigung die (5) vorausgehende Erzählung über das unzulässige Verhalten eines Messeverkäufers als Grund für die Schließung seines Messestandes nochmals in Kurzform thematisiert. Diese Interpretation paßt gut zu der Analyse, daß man Geschäftsschädigung als satzwertige Ellipse z.B. ersetzen könnte durch die explizitere Formulierung Das (was der Verkäufer gemacht hat) ist Geschäftsschädigung. In ähnlicher Weise lassen sich auch die Teiläußerungen in Zeile 30-31 und 32-33 aus dem in Abschnitt 2 diskutierten Gespräch als Ellipsen analysieren.

Unsere exemplarische Behandlung satzwertiger Ellipsen soll noch durch zwei interessante Beispieltypen ergänzt werden.

\section{(6) Jetzt den Hammer}

Ellipsen wie (6), die etwa durch gib mir zu expandieren sind, werden u.a. von Schwabe (1988) genauer diskutiert. Mit den bisher angeführten Beispielen hat auch (6) noch die Eigenschaft gemeinsam, daß aus den zugrundeliegenden Aussagen 
jeweils die wesentlichen Prädikationsteile explizit formuliert werden. Gibt es umgekehrt - so kann man sich fragen - auch Ellipsen, bei denen nur das Referenzobjekt genannt wird und die Prädikation fehlt? Eine Beantwortung dieser Frage führt offensichtlich zu einer neuen Einschätzung der Rolle von Vokativ-Nominalphrasen. Einerseits ist es bemerkenswert festzustellen, daß solche Phrasen tatsächlich frei verschiebbar sind (so läßt sich etwa vor und nach jedem Satz eines Textes die an einen Adressaten gerichtete Anrede Hans einfügen). Andererseits können mit einer VokativNominalphrase unterschiedliche Prädikationenen wie z.B. Paß auf/komm her/wehe dir verbunden sein, die in der mündlichen Kommunikation oft durch korrespondierende Intonationskonturen signalisiert werden. Zusammengefaßt zeigt die Diskussion des Falls 1a, daß der oben definierte Satzbegriff genügend allgemein ist, um die unterschiedlichen, mehr oder weniger expliziten Formen von Sachverhaltsdarstellungen zu erfassen. Dabei ist die Sprechweise von Ellipsen im Sinne von 'Auslassung' insofern unangemessen, als bei ihnen gleichermaßen autonome, wenn auch rudimentärere Formen von Sätzen vorliegen (vgl. Kindt, 1985).

Fall 1b: Das problematische Äußerungsstück A entspricht evtl. deshalb nicht den Standarderwartungen an Sätze, weil es Teile enthält, die sprachlich inkorrekt/ gestört sind. Eine derartige Störung kann allerdings innerhalb von A durch eine Reparatur behoben werden, und in diesem Sinne sprechen wir von satzinterner Reparatur. Die Untersuchung bzw. Einführung von Reparaturen als Gegenstand der Syntaxforschung wurde in jüngster Zeit insbesondere durch die Arbeiten von Schegloff (1979) und Levelt (1983) gefördert, ist aber noch mit erheblichen grundlagentheoretischen Problemen konfrontiert (vgl. Kindt und Laubenstein, 1991). Eine für unsere Diskussion wesentliche Einsicht der Reparaturtheorie besagt, daß die früher übliche Einschätzung reparierter Äußerungen als irregulär falsch ist. Beispielsweise müßte man nach der von Weber (1977: 44-49) vertretenen Position die in unserem Korpus von Reparaturen vorgefundene Äußerung

\section{(7) Also die die Grund die Grundform sind is nich is nich eckig sondern rund}

crst von ihren Störungen 'reinigen', also in

\section{(7a) Also die Grundform is nich eckig sondern rund}

überführen, bevor man die Äußerung für eine grammatische Analyse verwenden kann. Gegen diese Position steht die Erkenntnis, daß die Durchführung von Reparaturen - in (7) sind es drei sogenannte Covert-Reparaturen und eine syntaktische Reparatur - selbst grammatisch regulär sind. Diesbezüglich hat Levelt (1983) als erster auffällige Ähnlichkeiten zwischen Reparaturkonstruktionen und Koordinationsellipsen festgestellt, und ein von mir entwickelter Modellierungsansatz (vgl. Eikmeyer et al., 1994) gibt für zwei Grundtypen von Reparaturen genauer an, welche grammatischen Regeln in welcher Reihenfolge angewendet werden. Als Fazit für den Fall 1b läßt sich also sagen, daß satzintern durchgeführte Reparaturen den Status der Satzeinheit nicht tangieren. 
Fall Ic: Schließlich ist die Möglichkeit zu berücksichtigen, daß das Äußerungsstück A lokale Störungen enthält, die im Unterschied zu Fall lb nicht repariert werden. Die für die Beurteilung dieses Falls entscheidende Frage heißt: Wie wirkt sich eine unreparierte Störung auf die Korrektheitseinschätzung des Gesamttextes, in dem A vorkommt, aus? Für eine Diskussion dieser Frage wollen wir noch einmal den Gesprächsausschnitt von Abschnitt 2 betrachten. Dort gibt es vier Stellen mit unkorrigierten grammatischen Fehlern. So müßte nach üblicher Norm die Koordinationskonstruktion von Zeile 17/18 durch Einfügung von wer modifiziert werden zu und meinen damit wer weiß was erlangt zu haben. In Zeile 19/20 wäre bei daß einfache Leute sich auch ihre Worte finden das Reflexivpronomen sich zu tilgen, das möglicherweise noch aus einer ursprünglich geplanten Formulierung daß einfache Leute sich auch ihre Gedanken machen stammt. In Zeile 20/21 könnte bei und daß sind überhaupt nicht intellektuell zu sein braucht das Wort sind durch das ersetzt werden, und schließlich sollte in Zeile 22 die Reihenfolge von so und heute vertauscht werden, um eine korrekte Wortstellung zu erreichen (ich hab mich mit Herrn Frühbold heute so wunderbar über menschliche Probleme unterhalten).

Die genannten Inkorrektheiten können aufs Ganze gesehen als unerheblich gelten; zumindest beeinträchtigen sie die Einschätzung des gesamten Gesprächsausschnitts als korrekten Text nicht wesentlich, ${ }^{4}$ und dies ändert sich auch nicht durch die Anwendung des Verschiebetests. Die oben vorgeschlagene Satzdefinition führt also - wenn gewünscht - in folgendem Sinne zu einem 'liberalen' bzw. fehlertoleranten Satzbegriff: Äußerungen, die nur unwesentlich von korrekt gebildeten Sätzen abweichen, kann man selbst noch als Sätze einstufen. Auf diese Weise wird einerseits verhindert, daß Äußerungen mit geringfügigen Fehlern (was insbesondere in der gesprochenen Sprache zum Normalfall gehört) aus dem Gegenstandsbereich der Linguistik herausfallen. Andererseits ist gewährleistet, daß die Zerlegbarkeit sprachlich korrekter Texte in Sätze auch dann gilt, wenn das Korrektheitskriterium den menschlichen Wahrnehmungsgewohnheiten entsprechend so interpretiert wird, daß unwesentliche Inkorrektheiten einzelner Teile nicht auf das Gesamturteil durchschlagen. Welche Logik derartigen Teil-Ganze-Beurteilungen genauer zugrunde liegt, ist eine wissenschaftstheoretisch wichtige Frage, auf die ich hier nicht näher eingehen kann (vgl. Kindt, 1993d).

Unreparierte minimale Äußerungsstörungen werden von Kommunikationsteilnehmern so behandelt, als seien sie nicht geschehen; d.h. sie werden 'stillschweigend' repariert oder teilweise (wie beim Überlesen von Druckfehlern) gar nicht wahrgenommen. Empirisch bedeutet das, daß das Verarbeitungsresultat von Äußerungen mit solchen Störungen identisch ist mit dem Resultat korrespondierender ungestörter Äußerungen. In diesem Sinne sind dann gestörte und zụgehörige ungestörte Äußerungen äquivalent, und man darf sich darauf konzentrieren, den einfacheren, ungestörten Fall zu untersuchen. M.a.W. die übliche Forschungspraxis, daß bestimmte Störungen in den betrachteten Sprachdaten einfach ignoriert werden, ist legitim. Als zwei konkrete Beispiele dieser Praxis seien genannt: Geräusche, von denen münd-

4 Diese Einschätzung könnte noch durch geeignete empirische Tests überprüft werden. 
liche Äußerungen evtl. überlagert werden, sind vernachlässigbar, und Sätze, die einander partiell überlappen, dürfen entzerrt und so behandelt werden, als seien sie nacheinander geäußert worden. Insgesamt gesehen, wird also die Organisationseinheit des Satzes auch nicht durch unreparierte Störungen beeinträchtigt, solange sie im Rahmen des Normalen bleiben.

Nach den drei Fällen la-1c muß nun die Möglichkeit untersucht werden, daß das problematische Äußerungsstück A selbst nicht frei verschiebbar ist. Grund hierfür könnte sein, daß A entweder zum vorausgehenden Satz $S_{1}$ oder zum nachfolgenden $S_{a t} z_{2}$ gehört, bzw. daß bei einer geeigneten Aufteilung von $A$ in $A_{1}$ und $A_{2}$ die beiden Sequenzen $S_{1} \Lambda_{1}$ und $\Lambda_{2} S_{2}$ jeweils Sätze bilden. Denn im Sinne von Bloomfield (1926) bedeutet Nichtverschiebbarkeit gerade, daß bestimmte syntaktische Abhängigkeitsbeziehungen zwischen $A$ und $S_{1}$ oder $S_{2}$ bestehen. Insofern muß das mit $A$ zusammenhängende Segmentierunsproblem darauf beruhen, daß man die zu den betreffenden Abhängigkeitsbeziehungen gehörigen syntaktischen Konstruktionen nicht erkannt hat. Soweit ich sehe, sind als einschlägige Problemfälle nur Ellipsenund Reparaturkonstruktionen zu diskutieren.

Fall 2a: Üblicherweise unterscheidet man zwei Arten von syntaktisch kontextabhängigen Ellipsen: Koordinationsellipsen und Adjazenzellipsen (vgl. Klein, 1985). Diese Unterscheidung ist aber nicht trennscharf, weil bestimmte Adjazenzellipsen, nämlich die sogenannten parallelen Fortführungen, teilweise zu den Koordinationsellipsen gehören.

(8) A Ich komme heute abend

B Ich nicht

Von Klein (1985, 1993) werden derartige Beispiele zu den Adjazenzellipsen gezählt. Sie sind aber auch als asyndetische Koordinationsellipsen einstufbar, weil die Konjunktion und ohne Bedeutungsveränderung zwischen den beiden Konstruktionsteilen eingefügt werden kann. Tatsächlich hat man bei asyndetischen Koordinationskonstruktionen nicht die nach der Satzdefinition von Bloomfield notwendige Konsequenz gezogen und den elliptischen Konstruktionsteil als unselbständige Äußerung erkannt. Im Sinne dieser bzw. unserer obigen Satzdefinition bildet (8) aber als Ganzes einen (kooperativ produzierten) Satz. Dasselbe gilt generell für Adjazensellipsen und ist relativ zum vorverständlichen Satzbegriff und zur gängigen Interpunktion besonders überraschend bei Frage-Antwort-Ellipsen (vgl. Kindt, 1985: 185-186).

(9) Wer hat wem geschrieben? Mein Freund dem Kanzler und ich dem Bundespräsidenten.

Die beiden Ellipsenbeispiele (8) und (9) realisieren den Subfall, daß das problematische Äußerungsstück A syntaktisch noch zum vorausgehenden Satz $S_{1}$ gehört (anaphorische Ellipsen). Im Gegensatz zu Adjazenzellipsen ist man in der Grammatikforschung bei anaphorischen Koordinationsellipsen wie 
(10) Mein Freund schreibt heute einen Brief, (und) ich morgen.

von vornherein von einer syntaktischen Zusammengehörigkeit der Konstruktionsteile ausgegangen. Einstufungsschwierigkeiten gab es auch nicht im Subfall kataphorischer Ellipsen, bei denen das problematische Äußerungsstück A dem nachfolgenden Satz $\mathrm{S}_{2}$ zuzurechnen ist, weil dieser Ellipsentyp nur von Koordinationsellipsen realisiert wird.

(11) Eva parkt jetzt vor, Max parkt nachher neben der Garage.

Als kurzes Fazit für den Fall 2a der syntaktisch kontextabhängigen Ellipsen kann man festhalten, daß sie satzinterne Konstruktionen darstellen. Damit löst sich ein Problem, das Rath (1993: 469) am Beispiel von

(12) A Ja das genücht ja auch*

B Ja mir nicht $(P)^{*}$

diskutiert. Die Unterscheidung zweier Äußerungseinheiten in (12) widerspricht nicht der Organisationseinheit des Satzes, sondern bestätigt sie, weil die beiden Einheiten zu einem Satz zusammengefügt werden. Zugleich ist es unproblematisch, daß der Äußerungsbeitrag der Sprecherin B eine syntaktisch kleinere Einheit als die des Satzes realisiert. Genereller kann man bei der Untersuchung kooperativ untersuchter Sätze feststellen, daß es im Prinzip keine Einschränkungen dafür gibt, welche Bestandteile von wem produziert werden.

Fall 2b: Adjazenzellipsen wurden bisher auch nicht ausreichend gegenüber Reparaturkonstruktionen abgegrenzt, und so werden ihnen etwa bei Klein (1993) Korrekturen wie

\section{(13) Otto hat hundert Mark gewonnen. - Nein, Peter tausend verloren.}

zugerechnet. Mit dieser Abgrenzungsproblematik brauchen wir uns hier nicht näher zu beschäftigen. Wichtig ist aber, daß auch nicht frei verschiebbare Äußerungen, mit denen eine Reparatur des vorangegangenen Satzes durchgeführt wird, syntaktisch zu diesem Satz gehören. Genauer muß man zwei grammatisch unterschiedliche Typen von satzinternen Reparaturkonstruktionen unterscheiden, die ich Überbrückungsund Nachtragsreparaturen nenne (vgl. Eikmeyer et al., 1991; Kindt und Laubenstein, 1991). Die im obigen Beispiel (7) enthaltenen Reparaturen sind alle Überbrückungsreparaturen, während (13) eine Nachtragsreparatur realisiert.

Nachtragsreparaturen verhalten sich bezüglich der Satzsegmentierung genauso wie anaphorische Ellipsen, d.h. der Reparaturversuch/Nachtrag gehört satzmäßig zur vorhergehenden Äußerung. Überbrückungsreparaturen ähneln demgegenüber kataphorischen Ellipsen, und der grammatisch gestörte Beginn einer Äußerung ist satzmäßig dem nachfolgenden Äußerungsteil zuzurechnen. Dies ist unmittelbar einsichtig bei einer Überbrückungsreparatur wie 
(14) Und dann brauchen whabn wir bláue und da brauchen wir zwéi von den langen' dünnen,.

In (14) kann der gestörte Äußerungsbeginn und dann brauchen $w$ nicht abgetrennt zu werden, ohne die Integrität des Satzes insgesamt zu zerstören. Aber auch wenn eine solche Abtrennung möglich ist und die Reparaturdurchführung den gestörten Äußerungsbeginn konstruktionsmäßig in keiner Weise fortführt oder wiederholt, kann man dafür argumentieren, daß der Äußerungsbeginn syntaktisch zum nachfolgenden Satz gehört.

\section{(15) Ich hab doch d der blaue is doch nich so hoch wie der rote}

Satzabbrüche wie in (15) gehören zu den sogenannten Blockadereparaturen (vgl. Kindt und Laubenstein, 1991). Der Name deutet an, daß die Fortführung einer begonnenen Konstruktion aus irgendeinem Grunde blockiert ist und durch die Formulierung einer neuen Konstruktion ersetzt wird. Derselbe Fall liegt auch bei Satzabbrüchen vor, die durch Unterbrechung und Rederechtsübernahme durch einen Gesprächspartner verursacht sind. Die syntaktische Zusammengehörigkeit zwischen Äußerungsbeginn und Reparaturdurchführung besteht bei (15) ausschließlich in dem Effekt, daß die lokale syntaktische Störung neutralisiert wird. Der Neutralisationseffekt geht verloren, wenn man den Verschiebetest anwendet und den abgebrochenen Äußerungsbeginn ich hab doch $d$ an das (hier nicht dokumentierte) Textende rückt; dabei wird unterstellt, daß es für einen Text eine wesentliche Störung bedeutet, wenn er durch eine abgebrochene Äußerung beendet wird.

Wenn die eben vorgeschlagene syntaktische Behandlung von Satzabbrüchen Bestand hat, dann kann die in Abschnitt 2 formulierte These der vollständigen Zerlegbarkeit von korrekten Texten in Sätze als bestätigt gelten. Dabei war für die hier entwickelte Lösung der bekannten Segmentierungsprobleme wesentlich, daß die im Anschluß an Bloomfield definierte Satzdefinition einerseits die Inadäquatheit bestimmter alltagstheoretischer bzw. historisch tradierter Syntaxnormen und andererseits die Notwendigkeit einer Berücksichtigung einiger bisher nicht erfaßter satzinterner Konstruktionen deutlich gemacht hat. Insgesamt dürften damit die Ergebnisse von Abschnitt 3 und 4 den Status des Satzes als zentrale Organisationseinheit von Kommunikation ausreichend belegt haben. Deshalb müssen im folgenden nur noch bestimmte, vorwiegend wissenschaftstheoretische Fragen der Satzdefinition geklärt werden.

\section{Satzdefinition und Erklärungsanspruch von Grammatiken}

Die Satzdefinition von Abschnitt 4 hängt in noch zu klärender Weise vom Korrektheitskonzept ab. Sie macht aber - aus gleich zu erläuternden Gründen - keinen Gebrauch von Aussagen über Grammatikregeln. Damit unterscheidet sich ihr Ansatz prinzipiell von der häufig vertretenen Auffassung, daß es in einer Satzdefinition um eine grammatikabhängige Explikation des Satzbegriffs gehe, d.h. daß der 
Satzbegriff das zentrale Explikandum von Grammatikmodellen sei (vgl. z.B. Hundsnurscher, 1980: 212). Diese Auffassung stammt ursprünglich aus der Logik bzw. aus der Theorie formaler Sprachen, und für die dort zu erfüllenden Aufgaben ist es durchaus angemessen, Grammatiken, also syntaktische Regelsysteme, nur zur Satzdefinition zu verwenden. Wenn man in der Linguistik die Funktion von Grammatiken darauf reduziert, den intuitiven/vorverständlichen Satzbegriff angemessen zu explizieren, dann verzichtet man darauf, mit Grammatiken einen empirischen Erklärungsanspruch zu verbinden (vgl. Kindt, 1985: 185). Erklärungsstarke und prognosefähige empirische Theorien sollten demgegenüber folgende drei Aufgaben erfiillen. Zunächst muß man eine den Bereich der relevanten Untersuchungsobjekte eingrenzende empirisch operationalisierte Gegenstandsdefinition angeben. Anschließend stellt man Hypothesen über den Gegenstandsbereich auf und versucht, sie mit logischen und/oder empirischen Verfahren zu überprüfen (vgl. Kindt und Laubenstein, 1991: 12ff.). Angewendet auf die Syntax, besagt diese wissenschaftstheoretische Konzeption, daß man - wie in Abschnitt 4 vorgeführt - zuerst eine grammatikunabhängige Satzdefinition formuliert und danach Syntaxregeln als empirische Hypothesen über die Bildung von Sätzen aufstellt. Eine Bestätigung oder Falisifikation solcher Regeln ist ebenfalls nur möglich, wenn man für die von einem syntaktischen Regelsystem generierten sprachlichen Objekte grammatisch unabhängig entscheiden kann, ob sie Sätze bilden oder nicht. Der wesentliche Nachteil der obengenannten Explikationsauffassung liegt also darin, daß bei diesem Ansatz die empirische Adäquatheit einer Grammatik nur durch Vergleich der erzeugten sprachlichen Objekte mit den Sätzen im vorverständlichen Sinne beurteilt werden kann. Weil aber dann die Einstufung einer Äußerung als Satz nur intuitiv entscheidbar ist, lassen sich auch Anwendungsbereich und Erklärungsanspruch von Grammatiken nicht eindeutig überprüfen. Die konkreten negativen Auswirkungen einer fehlenden grammatikunabhängigen Satzdefinition haben wir im vorigen Abschnitt schon aufgezeigt, denn die bisherige Unklarheit über den syntaktischen Status von Ellipsen und Reparaturen basierte gerade maßgeblich auf dem Fehlen einer solchen Definition. Allerdings ist es aufgrund der Komplexität sprachlicher Entitäten in der Linguistik generell nicht einfach, adäquate Gegenstandsdefinitionen zu finden, und deshalb bildet die Problematik der Satzdefinition keinen Einzelfall. Unter den hier diskutierten Phänomenen ist auch die Explikation des Ellipsenbegriffs (vgl. Kindt, 1985; Günther et al., 1991) und des Reparaturbegriffs (Kindt und Laubenstein, 1991) ein schwieriges Unterfangen. Dabei besteht eine wesentliche Ursache für die zugrundeliegenden Schwierigkeiten darin, daß die klassische wissenschaftstheoretische Vorstellung geschlossener Gegenstandsdefinitionen in der Wissenschaftspraxis noch nicht abgelöst ist durch die in der Konzeption der Theoriendynamik (vgl. Sneed, 1971; Stegmüller, 1980) vorgesehene Möglichkeit offener Definitionen.

Nach der wissenschaftstheoretischen Begründung für die Zweckmäßigkeit einer grammatikunabhängigen Satzdefinition soll nun der Definitionsvorschlag aus Abschnitt 4 weiter präzisiert werden. 7unächst muß genauer zwischen den Aussagen "Die Äußerung A ist ein Satz" und "Die Äußerung A ist ein Satz des Textes T" unterschieden werden. 


\section{Modifizierte Satzdefinition:}

Eine Äußerung A ist ein Satz, wenn A am Anfang oder Ende jedes sprachlich korrekten Textes eingeführt werden kann, ohne daß dabei die Eigenschaft der Korrektheit verlorengeht.

Die Offenheit dieser Definition drückt sich darin aus, daß nur eine hinreichende Bedingung für das Vorliegen von Sätzen gegeben ist; es könnte also Sätze geben, die diese Bedingung nicht erfüllen. Zugleich ist eine absolute Verifikation der Bedingung nicht möglich, sondern nur eine induktive Stützung, die auf einer Überprüfung an endlich vielen, zufällig ausgewählten Texten beruht. Fine Verifizierbarkeit wäre aber gegeben, wenn man von vornherein eine endliche Menge von Texten als Bezugspunkt für den Einfügungstest auswählt.

$\mathrm{Daß}$ man in cincm Text ein Äußerungsstück A angeben kann, das im Sinnc unscrer Definition einen Satz bildet, ist noch nicht gleichbedeutend damit, daß A Satz des Textes ist. Hierzu ein Beispiel.

(16) Peter schreibt dem Bürgermeister einen Brief. Er fordert ihn auf, sich stärker für den Umweltschutz einzusetzen.

Das Äußerungsstück Peter schreibt dem Bürgermeister ist zwar ein Satz, aber kein Satz von (16). M.a.W. bei der Zerlegung von Texten in Sätze muß noch auf die Geltung einer Maximalitätsbedingung geachtet werden.

\section{Textbezogene Satzdefinition:}

Eine Äußerung A ist ein Satz des sprachlichen Textes T genau dann, wenn A ein Satz ist und wenn es zwei (evtl. leere) Texte $T_{1}, T_{2}$ derart gibt, daß sich $T$ zerlegen läßt in $T=T_{1} A T_{2}$.

Diese Definition läßt auch die Möglichkeit mehrdeutiger Segmentierungen zu, die wir in Abschnitt 3 diskutiert haben.

Ein bisher nicht thematisiertes Problem der Satzdefinition besteht in der Bezugnahme auf das Kriterium der sprachlichen Korrektheit. Im Rahmen der Reparaturtheorie (vgl. Kindt und Laubenstein, 1991) kann präzisiert werden, was unter sprachlicher Korrektheit zu verstehen ist und welche Typen von Korrektheitsproblemen voneinander unterschieden werden müssen. Zugleich darf man annehmen, daß Kommunikationsteilnehmer fähig sind, über die Korrektheit von Äußerungen zu urteilen, auch wenn sie die zugrundeliegenden Grammatikregeln nicht explizit verbalisieren können. Das bedeutet, daß eine von expliziten Grammatikmodellen unabhängige empirische Operationalisierung des Korrektheitsbegriffs möglich ist.

Ein gängiges Verfahren zur Ermittlung von Korrektheitsurteilen ist die Sprecherbefragung. Wenn dieses Verfahren als alleinige Methode angewendet wird, ist dies aus mehreren, bekannten Gründen nicht unproblematisch. Zunächst ist eine Anwendung dieses Verfahrens nur möglich, wenn man mit den vorgesehenen Informanten über einschlägige Beurteilungsaspekte ihrer Sprache kommunizieren kann. Sofern diese Voraussetzung erfüllt ist, tritt das Problem auf, welche Fragen zur Beurteilung 
einer Äußerung/eines Textes man an die Informanten richten soll. Wenn etwa die Frage "Kann man das so in Ihrer Sprache sagen?"' mit "Ja'" beantwortet wird, dann hat man vielleicht einen brauchbaren Befund erhalten. Falls die Frage aber verneint wird, kann dies unterschiedliche Gründe haben, die nicht ausschließlich mit einer sprachlichen Inkorrektheit der Äußerung zusammenhängen müssen, sondern auch auf einer mangelnden semantischen oder pragmatischen Akzeptabilität beruhen können. Als Lösung dieses Problems bietet sich an, daß man Situationen konstruiert, in denen die semantische/pragmatische Akzeptanz der betreffenden Äußerung erhöht wird und auf diese Weise eine Differenzierung von Inakzeptabilitätsurteilen erreicht. Dieses Verfahren ist auch speziell bei Durchführung des Verschiebetests zweckmäßig. Wenn man beispielsweise die beiden Sätze in (16) vertauscht, dann erhält man einen ziemlich inakzeptabel wirkenden Text. Dieser Eindruck ändert sich bei Konstruktion einer Situation, in der die beiden Personalpronomina z.B. auf zwei weitere Personen referieren. Zumindest wird dann deutlicher, daß die Inakzeptabilität nicht durch grammatische Inkorrektheit verursacht ist.

Die Offenheit der Satzdefinition läßt aber auch noch eine andere Argumentation zu. Wenn man in (16) beispielsweise Adam für er und den Gemeindedirektor für ihn einsetzt, dann erhält man bei Anwendung des Verschiebetests einen Text, der genauso akzeptabel ist wie (16). Dieses Resultat und die Ergebnisse anderer, ähnlicher Verschiebeproben legitimieren die Einstufung des durch die beiden Substitutionen modifizierten zweiten Textteils von (16). Aufgrund der strukturellen Verwandtschaft zum modifizierten Textteil läßt sich dann auch der unmodifizierte Teil als Satz einstufen. Genauer gesagt, führt man eine weitere hinreichende Definitionsbedingung für die Identifizierung von Sätzen ein, die allerdings vergleichsweise stärker theorieabhängig ist, weil für die Überprüfung von Aussagen über die strukturelle Verwandtschaft von Äußerungen bestimmte grammatiktheoretische Grundlagen mit zugehörigen Testverfahren erforderlich sind. Außerdem kann man mit Hilfe des Verwandtschaftskriteriums erst dann Äußerungen als Sätze identifizieren, wenn zuvor anderen Äußerungen mit Hilfe des Verschiebbarkeitskriteriums der Satzstatus zuerkannt wurde und wenn man für den Bereich der so ermittelbaren Sätze bereits die erforderliche Strukturtheorie entwickelt hat. Der so skizzierte Prozeßcharakter von aufeinanderfolgenden Definitions- und Theoriebildungsschritten macht gerade den dynamischen Aspekt der modernen Konzeption der Wissenschaftstheorie aus.

Die Reichweite der durch den Permutationstest operationalisierten Definitionsbedingung endet generell bei Äußerungen, die in bestimmten textgrammatischen Abhängigkeitsbeziehungen zu ihren Nachbaräußerungen im Text stehen. In solchen Fällen ist der Satzstatus grundsätzlich nur über das Kriterium der Strukturverwandtschaft ermittelbar. Dies soll noch einmal an folgendem Beispiel veranschaulicht werden.

(17a) Zuerst geht Gert einkaufen. Danach fährt Hans in die Universität.

(17b) *Danach fährt Hans in die Universität. Zuerst geht Gert einkaufen.

(17c) Jetzt geht Gert einkaufen. Nachher fährt Hans in die Universität.

(17d) Nachher fährt Hans in die Universität. Jetzt geht Gert einkaufen. 
Die in Informantenbefragungen erhobenen Korrektheitsurteile sind noch mit einem weiteren Problem verbunden. Wölck (1980) schilderı sehr anschaulich, daß Sprecher ihren Urteilen eventuell alltagstheoretische Sprachnormen zugrunde legen, die sie in ihrer eigenen Kommunikationspraxis nicht befolgen. Insofern müssen die Befunde aus Informantenbefragungen kontrolliert und ergänzt werden durch die Ergebnisse von Korpuserhebungen und deren Analyse. Dabei kommt es speziell für die Ziele von Syntaxforschung darauf an, daß man neben vorgefundenen oder in spontaner Kommunikation aufgenommenen Texten auch Äußerungen unter spezifisch variierten Situationsbedingungen elizitiert. Die entsprechenden Erhebungsverfahren will ich jetzt aber nicht diskutieren. Wichtig ist in unserem Diskussionszusammenhang nur, daß über die Rekurrenzeigenschaft, d.h. bei häufigerem Vorkommen eines Text-/Äußerungsteils im Korpus induktiv auf seine Korrektheit geschlossen werden kann. Wenn sich auf diese Weise verschiedene Äußerungsteile als korrekt ergeben haben und wenn die bisher entwikelte Theorie schon einschlägige Verknüpfungsregeln enthält, dann lassen sich auch Korrektheitsaussagen für komplexere Äußerungen ableiten. Die Inkorrektheit eines (potentiellen) Äußerungsteils kann nur unter besonderen Bedingungen aus seinem Nichtvorkommen im Korpus erschlossen werden; direkt belegbar ist sie aber, wenn man im Korpus eine explizite sprachliche Korrektur des Äußerungsteils vorfindet.

Insgesamt gesehen dürfte also deutlich geworden sein, daß die in der Satzdefinition enthaltene Korrektheitsbedingung ausreichend empirisch verankert ist und die Definition somit eine grammatikunabhängige Identifizierung von Sätzen erlaubt.

\section{Literaturangaben}

Betten, Anne, 1976. Ellipsen, Anakoluthe und Parenthesen. Fälle für Grammatik, Stilistik, Sprechakttheorie oder Konversationsanalyse? In: Deutsche Sprache 3, 207-230.

Bloomfield, Leonard, 1926. A set of postulates for the science of language. Language 2, $153-164$.

Eikmeyer, Hans-Jürgen, Walther Kindt, Uwe Laubenstein, Sebastian Lisken, Thomas Polzin, Hannes Rieser und Ulrich Schade, 1991. Kohärenzkonstitution im gesprochenen Deutsch. In: Gert Rickheit (Hrsg.), Kohärenzprozesse, 59-136. Opladen: Westdeutscher Verlag.

Eikmeyer, Hans-Jürgen, Walther Kindt, Uwe Laubenstein, Sebastian Lisken, Hannes Rieser und Ulrich Schade, 1994. Coherence regained. In: Christopher Habel, Gert Rickheit (Hrsg.), Focus and coherence in discourse processes. Berlin: de Gruyter.

Fiehler, Reinhard, 1992. Analyse und Beschreibungskategorien für geschriebene und gesprochene Sprache. Alles eins? Ms., Universität Bielefeld.

Gülich, Elisabeth, 1970. Makrosyntax der Gliederungssignale im gesprochenen Französisch. München: Fink.

Günther, Udo, Walther Kindt, Uwe Schade, Lorenz Sichelschmidt und Hans Strohner, 1991. Elliptische Koordination. KoLiBri-Arbeitsbericht 32, DFG-Forschergruppe Kohärenz. Universität Bielefeld. Überarbeitete Fassung in: Linguistische Berichte 146, 312-342 (1993).

Heringer, Hans Jürgen, 1970. Einige Ergebnisse und Probleme der Dependenzgrammatik. Der Deutschunterricht 22, 42-98.

Hoffmann, Ludger (Hrsg.), 1992. Deutsche Syntax. Berlin: de Gruyter.

Hundsnurscher, Franz, 1980. Syntax. In: Hans Peter Althaus, Helmut Henne, Herbert Ernst Wiegand (Hrsg.), Lexikon der Germanistischen Linguistik. 2. Aufl. Tübingen: Niemeyer.

Kindt, Walther, 1985. Grammatische Prinzipien sogenannter Ellipsen und ein neues Syntaxmodell. In: Reinhard Meyer-Hermann, Hannes Rieser (Hrsg.), 161-290. 
Kindt, Walther, 1991. Informationsdynamik bei der grammatischen Verarbeitung. KoLiBri-Arbeitsbericht 36, DFG-Forschergruppe Kohärenz. Universität Bielefeld.

Kindt, Walther, 1993a. Struktur, Funktion und Dynamik von Erzählungen. In: Johannes Janota (Hrsg.), Vielfalt der kulturellen Systeme und Stile. Vorträge des Augsburger Germanistentags 1991, Bd. 1, 151-166. Tübingen: Niemeyer.

Kindt, Walther, 1993b. Die Modellierung von Nichtmonotonie im Rahmen der Klassischen Logik. Ms., Universität Bielefeld.

Kindt, Walther, 1993c. Wortstellung als Problem einer Dynamischen Grammatik. In: Brigitta Haftka (Hrsg.), Was determiniert Wortstellungsvariation, 49-62. Opladen: Westdeutscher Verlag.

Kindt, Walther, 1993d. Teil-Ganze-Schlüsse. Ms., Universität Bielefeld.

Kindt, Walther und Uwe Laubenstein, 1991. Reparaturen und Koordinationsstrukturen. KoLiBriArbcitsbericht 20, DFG-Forschergruppe Kohärenz. Universität Bielefeld.

Klein, Wolfgang, 1985. Ellipse, Fokusgliederung und thematischer Stand. In: Reinhard Meyer-Hermann, Hannes Rieser (Hrsg.), 1-24.

Klein, Wolfgang, 1991. Ellipse. In: Joachim Jacobs, Arnim v. Stechow, Wolfgang Sternefeld, Theo Vennemann (Hrsg.), Syntax. An international handbook of contemporary research. Berlin: de Gruyter.

Langer, Inghard, Friedemann Schulz von Thun und Reinhard Tausch, 1974. Verständlichkeit in Schule, Verwaltung, Politik, Wissenschaft. München: Reinhardt.

Levelt, Willem J.M., 1983. Monitoring and self-repair in speech. Cognition 14, 41-104.

Meyer-Hermann, Reinhard und Hans Rieser (Hrsg.), 1985. Ellipsen und fragmentarische Ausdrücke. Bd. 1. Tübingen: Niemeyer.

Rath, Rainer, 1976. Gesprochenes Deutsch und seine Gliederung. Bciträge zu den Fortbildungskursen des Goethe-Instituts für ausländische Lehrer an Schulen und Hochschulen. Goethe Institut München.

Rath, Rainer, 1979. Kommunikationspraxis. Göttingen: Vandenhoeck and Ruprecht.

Rath, Rainer, 1985. Geschriehene und gesprochene Form der heutigen Standardsprache. In: Werner Besch, O. Reichmann, S. Sonderegger (Hrsg.), Sprachgeschichte. Einn Handbuch zur Geschichte der deutschen Sprache und ihrer Erforschung, 2. Halbband, 1651-1663. Berlin: de Gruyter.

Rath, Rainer, 1992. Sprechen wir in Sätzen? Über Einheitenbildung im Alltagsdialog. In: Peter Suchsland (Hrsg.), Biologische und soziale Grundlagen der Sprache, 249-263. Tübingen: Niemeyer.

Rath, Rainer, 1993. Äußerungseinheiten als Bedeutungseinheiten. In: Gerhard Bartels, Inge Pohl (Hrsg.), Wortschatz-Satz-Text, 459-472. Frankfurt a.M.: Lang.

Schegloff, Emanuel A., 1979. The relevance of repair to synlix-for-conversation. In: Talmy Givón (Hrsg.), Syntax and semantics, Vol. 12: 261-286. New York: Academic Press.

Schwabe, Kerstin, 1988. Satzartige situative Ellipsen, ihre syntaktische und ihre semantische Repräsentation und ihre pragmatische Interpretation. Zeitschrift für Phonetik, Sprachwissenschaft und Kommunikationsforschung 41, 592-617.

Seidel, Eugen, 1935. Geschichte und Kritik der wichtigsten Satzdefinitionen. Jenaer Germanistische Forschungen 27, 114ff.

Sneed, Joseph D., 1971. The logical structure of mathematical physics. Dordrecht: Reidel.

Stegmüller, Wolfgang, 1980. Neue Wege der Wissenschaftsphilosophie. Berlin: Springer.

Texte deutscher gesprochener Standardsprache I 1971. München: Hueber.

Weber, Heinrich, 1977. Kleine generative Grammatik des Deutschen. Tübingen: Niemeyer.

Wölck, Wolfgang, 1980. Zuverlässigkeitskriterien für die Beschreibung von Sprechsprachen. In: Danièle Clement (Hrsg.), Empirische Rechtfertigung von Syntaxen, 22-37. Bonn: Bouvier. 